\title{
IMPLEMENTATION OF VLAN VIA WIRELESS NETWORKS USING OPNET MODELER
}

\author{
Tareq Al-Khraishi and Muhannad Quwaider \\ Department of Computer Engineering, Jordan University of Science and \\ Technology, Irbid, Jordan
}

\begin{abstract}
A VLAN is a logical rather than physical connection that allows grouping hosts together in the same broadcast domain, so that packets are only delivered to ports that are combined to the same VLAN. By characteristic VLAN network, we can improve efficiency of wireless network and save bandwidth. Furthermore, implementing VLAN greatly improves wireless network security by decreasing the number of hosts that receive copies of frames broadcasted by switches, so hosts holding critical data are kept on a separate VLAN. This paper compares wireless network with wireless network having VLAN deployment. The proposed Network is evaluated in terms of average throughput and delay using file transfer in heavy traffic and web browsing applications. The simulation was carried out by employing OPNET 14.5 modeler simulation and the results show that the use of VLAN via wireless network had improved the performance by decreasing the traffic resulting in minimizing delay time. In addition, implementing VLAN reduces the network throughput because the traffic that is received and forwarded has a positive relationship with throughput. Furthermore, we investigated to improve the throughput in a wireless VLAN network by using adhoc routing protocols. Evaluation, comparison of broad adhoc routing protocols like AODV, DSR, OLSR, TORA and GPR are conducted in order to show the effect of the proposed VLAN on the performance results, like throughput and delay.
\end{abstract}

\section{KEYWORDS}

WLAN, OPNET, AODV, Throughput, VLAN, Routing Protocols, Access Point.

\section{INTRODUCTION}

WLAN (Wireless Local Area Networks) allows devices, flexibility to move within a network from one place to another and connect to the LAN wirelessly via radio transmission. Wireless technology allows users to share data and applications and other resources, without being tied to connections [1][2]. Technically Wi-Fi (Wireless Fidelity) is referred as the communications standard for WLAN. Nowadays, one of the most common wireless technologies uses to transfer data, is the IEEE 802.11 standard, due to the need for high speed data rates many standards developed to meet customer's needs [3][4]. The table 1 . Show most commonly used protocol in today's environment [5]. 
Table 1. Summary of Various WLAN Standards.

\begin{tabular}{|c|c|c|c|}
\hline Standards & RF Band & Max. Data Rate & Range \\
\hline IEEE 802.11 & $2.4 \mathrm{GHz}$ & $2 \mathrm{Mbs}$ & $50-100 \mathrm{~m}$ \\
\hline IEEE 802.11b & $2.4 \mathrm{GHz}$ & $11 \mathrm{Mbps}$ & $50-100 \mathrm{~m}$ \\
\hline IEEE 802.11a & $5 \mathrm{GHz}$ & $54 \mathrm{Mbps}$ & $50-100 \mathrm{~m}$ \\
\hline IEEE 802.11g & $2.4 \mathrm{GHz}$ & $54 \mathrm{Mbps}$ & $50-100 \mathrm{~m}$ \\
\hline
\end{tabular}

Infrastructure WLAN as shown in Figure 1, consists of wireless stations and access points (AP), and support many wireless stations depending on the specification of the AP. A wireless network can connect to the Ethernet network by using UTP cable up to 100 meters from the AP to the hub or switch. Different access points having different locations and a confined communication radius [6]. The mobile stations can move while it is communicating and will automatically search and connect to the access point device using SSID (Service Set Identifier) which is a unique name of a wireless network matching with the AP is defined in the program, the SSID keeps the packets within the correct WLAN [1].

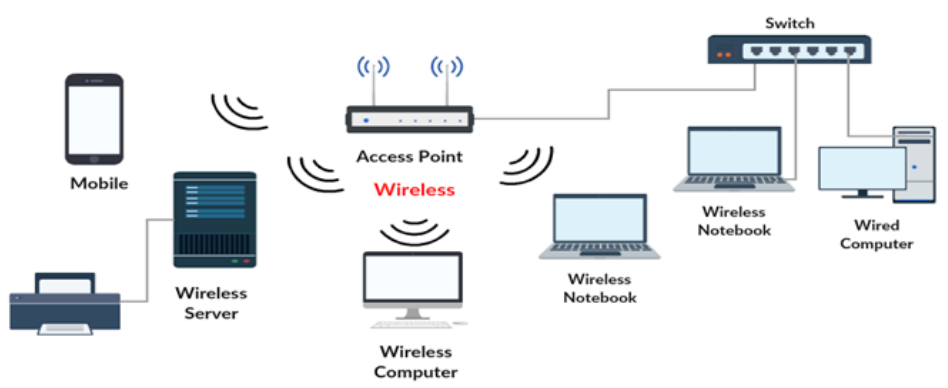

Figure 1. Wireless infrastructure diagram

There are many key performance metrics to consider when evaluating a wireless 802.11 WLAN solution, in this paper, we considered throughput and delay, throughput is the rate at which data is received successfully by the wireless LAN destination while delay is the time it takes to successfully deliver data from the source up to the destination node [6][7][8].

A Local Area Network (LAN) is usually defined as a broadcast domain which means that all connected devices are in the same physical LAN can communicate without the need for a router [7]. Virtual LANs (VLANs) which regularly defined as a set of devices on different physical LAN segments which can communicate as if they have a common LAN segment. Switches using VLANs to divide the network into separate broadcast domains without having the latency problems [8]. Trunk of VLAN used when connected switch's that support multiple VLANs together crossing the same Ethernet links as shown in Figure 2, the switches tag each frame sent between switches to distinguish which VLAN the frame belongs to. VLANs is based on the IEEE 802.1Q standard that defines the frame format for tagging [7][9]. 


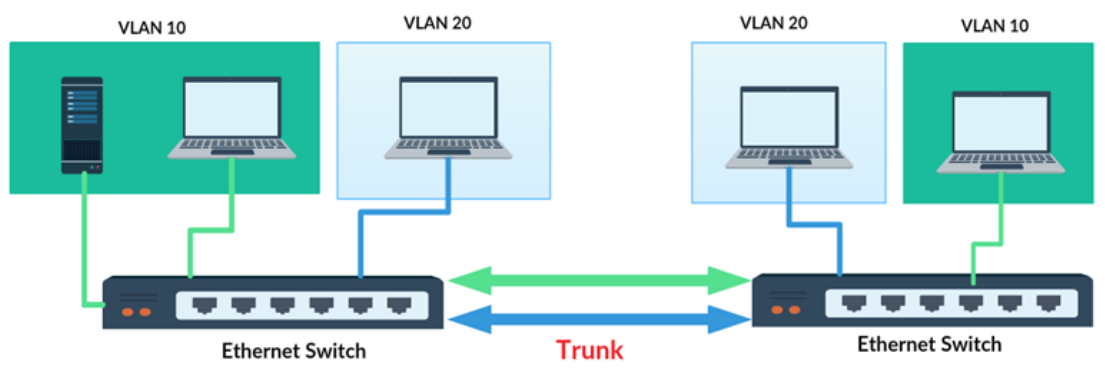

Figure 2. Network with Two VLANs

connection created for a specific purpose where hosts are enabled to send data directly to other hosts rather than going through an access point [13]. VLANs provide several Benefits, such as ease of administration, reduced broadcast traffic, and enforcement of security policies [8][9].

The Main Benefits of VLAN are listed below:

- Performance: The network traffic is full of broadcast. VLAN segment the large broadcast domain in to small broadcast domain which reduced unnecessary traffic in the network by sending packets only between ports that are combined to the same VLAN which reduce overhead and delay in addition to save the bandwidth.

- Organization: VLANs can be very useful to group the hosts logically according to their departments or jobs which are easy to handle as compared to a bigger broadcast domain.

- Security: In the same network, sensitive data can be broadcast which can be accessed by the outsider, but by creating VLAN, greatly enhance network security by reducing the number of hosts that receive copies of frames which the switches are broadcast and keep hosts that hold sensitive data on a separate VLAN.

- Cost reduction: VLANs can be used to create broadcast domains which less cost than expensive routers.

The 802.11 communications standard defines two operating modes [10]:

Infrastructure mode and adhoc mode. In infrastructure mode as shown in Figure 3, wireless hosts can communicate with each other via access point which is generally the default mode [6]. In adhoc mode as shown in Figure 4, wireless hosts can communicate with each other directly without AP as a peer-to-peer network form, every host acts as both a client and an access point at the same time. An adhoc network is a temporary network

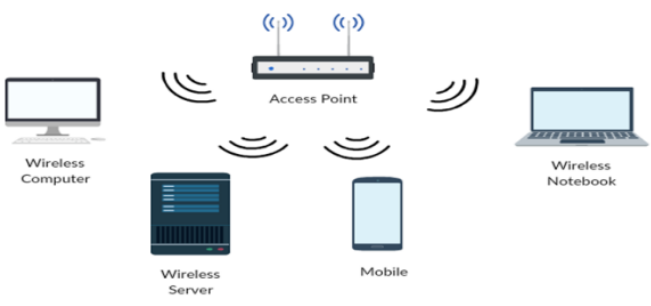

Figure 3. The 802.11 network Infrastructure operating modes 


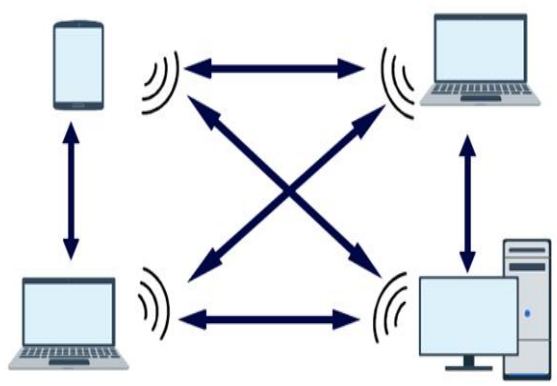

Figure 4. The 802.11 network adhoc operating modes

This paper provides huge potential for improving the performance of the wireless network. This section provides a brief introduction to wireless network and a virtual local area network (VLAN), while the rest of the paper is organized as follows: Section 2 sheds lights on adhoc routing protocols with a focus on flat routing protocol classifications (reactive, proactive, and hybrid); Section 3 of the paper gives an briefly reviews of related work in wireless networks and VLAN networks in addition to most relevant previous studies concerning adhoc routing performance ; subsequently, simulation model describes the simulation tool, simulation performance metrics, simulation setup and scenarios will be implemented that presents three type of networks :wireless network, wireless network with VLAN and wireless VLAN network with adhoc routing protocols are given in section 4 . The section 5 shows the simulation results of a comparison between all the simulations done to show the evaluated of the performance metrics. The simulation of the scenarios design are carried out using OPNET. Eventually, the conclusion based on the entire work done and results evaluated is given in Section 6.

\section{Adhoc Routing Protocols Overview}

The routing protocols that have been developed for adhoc networks are playing an important role in affecting data transmission and the performance of the network. Since all nodes of networks behave as routers and take part in the discovery and maintenance of routes to other nodes in the network, each routing protocol has its own routing strategy to decide the best path between the nodes. Routing protocols for adhoc networks can be classified into three main types: Proactive routing protocols, Reactive routing protocols, and Hybrid routing protocols [13] [17].

Proactive routing is also known as table driven routing protocols because each node in the network has one or more routes to any destination in its own routing table at any time [14]. Reactive routing protocols are also known as On-demand routing protocols, which are the source node create routes only when has data to send, if a route does not exist the protocol initiates a route discovery process to find a path to the destination [17]. In hybrid routing protocols, every node act reactively, in the region close to its and proactively outside of that region [14] [13].

In this section, a brief overview of the routing operations performed by the familiar protocols OLSR, GRP, DSR, AODV and TORA are discussed. Figure 5 illustrates these routing protocols that considered in this paper. 


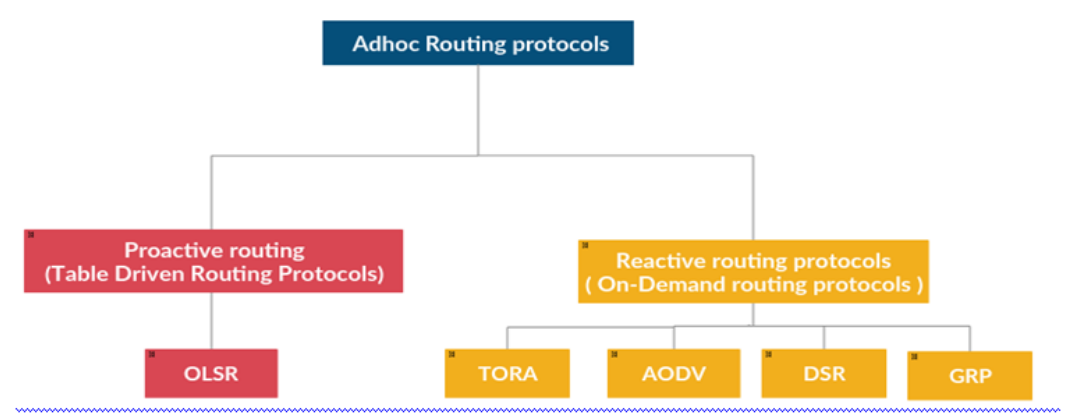

Figure 5. The adhoc routing protocols

Optimized Link State Routing (OLSR): Is a proactive protocol, which based on the link state algorithm [18]. The main concept of the protocol is to modify the changes of the network without creating control messages overhead. Thus, only a group of nodes, named Multi point Relays in the network responsible for broadcasting control messages and creating link state information. Every MPR chooses to broadcast link state information only between itself and the nodes that have selected it.

Adhoc On-Demand Distance Vector Routing (AODV): Is reducing the number of broadcasts by creating routes to the destination only when needed since nodes that are not on a selected path do not maintain routing information or participate in routing table exchanges [14]. When a source node has data to send and does not have a valid route to that destination, it initiates a path discovery process to find the destination node [15] [19].

Dynamic Source Routing (DSR): Is a simple and efficient routing protocol composed of two mechanisms, route discovery and route maintenance [14]. The source node uses route discovery to find a route when a request arrives and inserts the discovered routes in the packet header. Intermediate nodes do not need to maintain up-to-date routing information apart from participation in the path route discovery and maintenance [18] [15]. Temporally Ordered Routing Algorithm (TORA): Is an on-demand routing protocol [16]. The main objective of TORA is to limit control message in the highly dynamic mobile environment. Each node must initiate a query when it needs to send data to the destination. TORA essentially performs three tasks: Creation of a route from a source to a destination, maintenance of the route and erasure of the route when the route is not valid. Geographic Routing Protocol (GRP): Is classified as proactive routing protocol [17]. In GRP a source node collects network information by using the Global Positioning System (GPS) with a small amount of control overheads. Source node able to find routes and transmit data even if the current route is disconnected.

\section{RELATED WORKS}

VLAN can be used to form logical grouped hosts in the same domain, to reduce broadcast traffic and to enhance the security of networks. Hence, it offers benefits in terms of efficient use of bandwidth, performance and security. There exists a many author of research on wireless and VLAN network. The authors in [4] [10] [11] and [12] analyzed and evaluated the effect of the amount of traffic in a wireless network with the help of OPNET Modeler and using the main performance metrics such as throughput, average delay and load data. The authors concluded that, the delay time depending on the amount of traffic load it has. The higher load has greater delay time.

The author [7][8] suggest that LAN network performance can be improved by using VLANs, this done by analyze and evaluate the performance of LAN and VLAN networks and measuring 
key performance indicators. The results shown that increasing the number of VLANs will reduce the traffic rapidly.

In a wireless adhoc network, each node cooperates to maintain the network topology and packet transmission. We introduce the most relevant previous studies concerning adhoc routing performance.

The authors [13] [17] compare the performance of two on-demand routing protocols for ad hoc networks DSR and AODV. The simulation results show that in a network with a small number of nodes DSR is more effective while AODV is more effective for higher number of nodes

Another work [14] [15] compares performances of proactive and reactive protocols DSDV, DSR and AODV based on three performance metrics: throughput, average delay and packet delivery ratio by using the NS-2 simulator. The authors conclude, DSR performs better performance because it has less routing overhead considering the above said three metrics. The authors [18] evaluated four adhoc routing protocols: AODV, DSR, TORA and OLSR. Throughput and delay were used as performance metrics. Simulation results reveal that are evaluated under different scenarios using Hypertext Transfer Protocol traffic. The overall results show that the OLSR performs better in terms of throughput and delay than the reactive routing protocols DSR, AODV and TORA for a moderate number of nodes in the network.

As we can see in this section the most relevant previous studies concerning about wireless LAN, VLAN and adhoc routing performance separately. Our work differs in that we proposed employment of Virtual Local Area Network (VLAN) over a wireless LAN network which can effectively confine the broadcast traffic and prevent bandwidth wastage, as well as enforcement of security policies which is a drawback inherent to the traditional switched used in wireless networks. Reduced throughput is the major drawback of using VLAN since both short delay and high throughput are desirable in wireless networks, so we investigate to solve this problem and improve the wireless VLAN network throughput performance via the use the most widespread protocols for adhoc networks.

\section{Simulation Model Description}

\subsection{Simulation Tool:}

Simulation design and analysis are done on OPNET (Optimized Network Engineering Tools) 14.5 simulator, which provides a real network scenario design, implementation and gathering of results using different metrics. This paper concern about the evaluation of employment VLAN over wireless networks and examine the impact of using five adhoc routing protocols AODV, OLSR, DSR, TORA and GRP to improve the wireless VLAN network throughput performance.

\subsection{Simulation performance metrics:}

The following two important performance metrics are considered for evaluation the network in this paper:

- Throughput: Also known as packet delivery ratio, it is the average rate of data packets transmitted and received successfully by every node in the network. It is measured in bits per second. In wireless networks, a better performance is indicated by a higher value of throughput.

- Delay: It is the time takes for a packet to be transmitted from the source node to the destination. It is measured in seconds; lower value delay reflects better performance. 


\subsection{Simulation setup:}

The simulation setup Initially investigates the WLAN model with and without VLAN to study the performance of add VLAN in to the wireless network. The setup assumes that there are two servers and two switches that connect two sections. The simulation models run with 20 nodes, which randomly placed within a square area of $1000 \mathrm{~m} \times 1000 \mathrm{~m}$, each five nodes connected wirelessly to Access Point using unique BSS. The MAC protocol used to be the IEEE 802.11b, which support wireless communication at a rate of up to 11Mbps [19]. Nodes (PC1-PC5) connected wirelessly to Access Point 1, (PC11-PC15) connected wirelessly to Access Point 4, (PC6-PC10) connected wirelessly to Access Point 2 and (PC16-PC20) connected wirelessly to the Access Point 3 as shown in Figure 6.

The access points 1 and 3 are connected to the switch 1 by 100Base-T in addition to access point 2 and 4 is connected to the switch 2 by 100Base-T. Ethernet Server_1 connected by 100Base-T to Switch 1 and Ethernet server_2 connected by 100Base-T to Switch 2.

A heavier application traffic flow in the network was generated, which each node will be processing from the respective application two Ethernet servers in the network. The application traffic generated was as, File Transfer Protocol (FTP) application and Hyper Text Transfer Protocol (HTTP) applications. Furthermore, we also need to define applications and profiles by adding a node for each, then associate the workstation with the profiles.

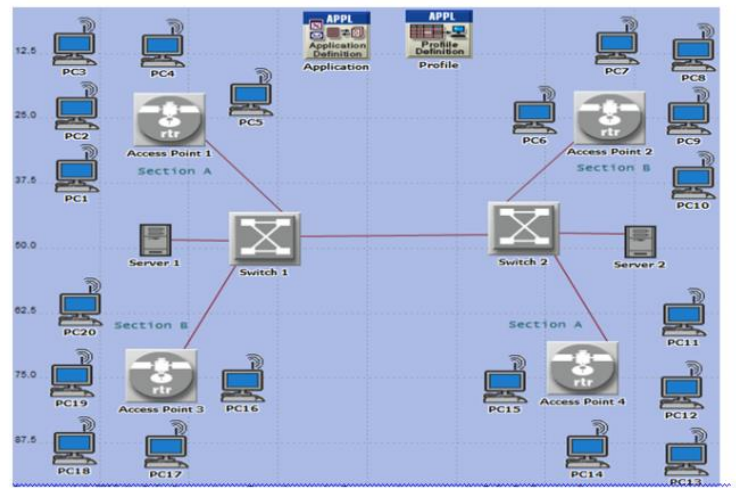

Figure 6. The Proposed wireless LAN network with 20 workstations

\subsection{Simulation Scenarios:}

We have considered a square area of $1000 \mathrm{~m} \times 1000 \mathrm{~m}$ for our simulation study purpose here, three scenarios have been created:

The first scenario (Wireless network without VLAN): This scenario considered by a wireless network as shown in Figure 6. We put one Access Point (AP) for each section to transmit wireless signals. All APs is connected by two Ethernet switches and there are two Ethernet servers that provide applications used for the workstations, in this scenario, there is one broadcasting domain in the network, so any workstations can communicate with two servers which increased the throughput of the wireless network. Wireless LAN delay and throughput are analyzed to monitor the performance.

A second scenario (Wireless network with VLAN): In this scenario, implemented VLAN to the wireless network so the network is divided into two VLANs (VLAN10, VLAN20) as shown in Figure7. VLAN 10 consists of 10 workstations (PC1-PC5) connected wirelessly to Access Point 1 and (PC11-PC15) connected wirelessly to Access Point 4 in addition to Ethernet server_1 
while VLAN 20 consists of 10 workstations (PC6-PC10) connected wirelessly to Access Point 2 and (PC16-PC20) connected wirelessly to Access Point 3 in addition to Ethernet server_2. Trunk of VLAN used when connected switches because they support multiple VLANs together crossing the 100base-T Ethernet links. Wireless LAN delay and throughput are analyzed to monitor the performance.

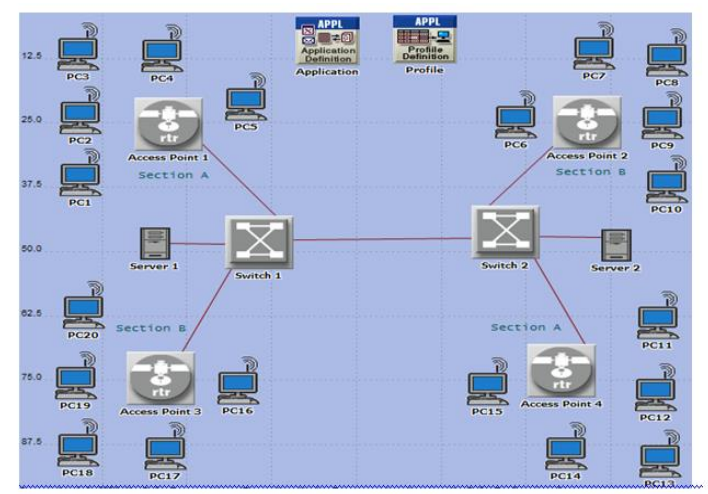

Figure7. The Proposed wireless LAN network with two VLANs

The third scenario (Using routing protocols to improve the performance of Wireless VLAN network): This scenario focused on a examine the impact of using five adhoc routing protocols AODV, OLSR, DSR, TORA and GRP to improve the wireless VLAN network throughput performance. Three types of network scenarios are designed: high density, medium density and low-density networks show the effect of increase the number of workstations on the performance of wireless network employing VLAN. The low-density network consists of 10 workstations in each VLAN, medium density network consists of 20 workstations in each VLAN and high-density network consists of 30 workstations in each VLAN. Wireless LAN delay and throughput are analyzed to monitor the performance.

\section{Simulation Results ANd Discussions}

The network has been executed with wireless network and wireless network with VLAN scenarios. The simulation parameters which have been considered for doing the performance comparison of the two scenarios are throughput and average delay. The simulation results are obtained by using OPNET 14.5 modeler.

In terms of the wireless delay observe levels of performance in VLAN via wireless network comparing with wireless network with no VLAN, as shown in Figure 8. Performance of these two networks remains very steady over the whole simulation period after the initial setup phase. However, the traffic has highly reduced by using VLAN technology because the network broadcasting domain has divided which mitigates the load in the network. In fact, VLAN via WLAN improved the performance by proved less wireless delay time. 


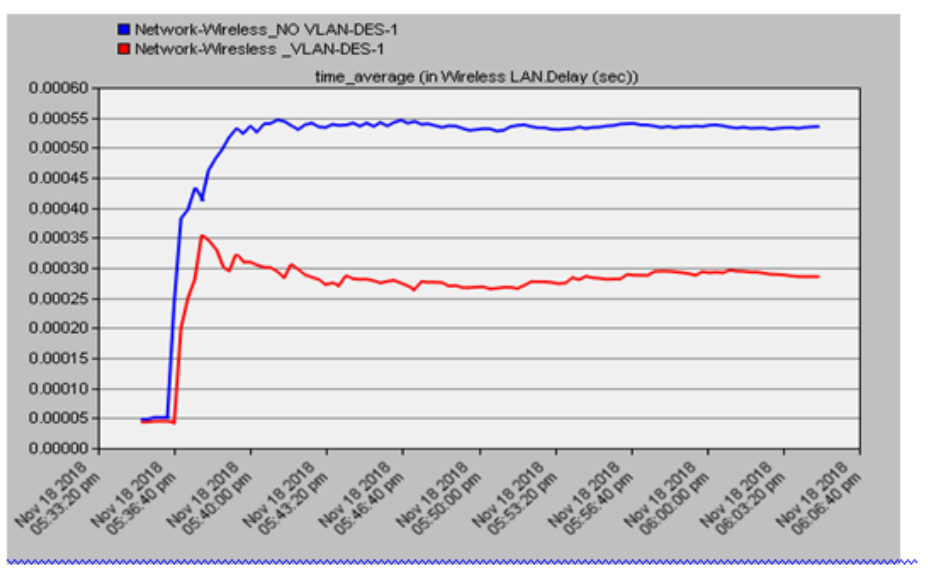

Figure 8 . The wireless LAN delay in the scenario 1 and scenario 2

In terms of the wireless throughput it found that VLAN via wireless has less throughput compared to WLAN scenario as shown in Figure 9. This is because the wireless network without VLAN scenario has higher traffic than VLAN via wireless scenario due to a single broadcast domain. Performance of these two networks almost remains steady over the whole simulation period band after the network has converged. The traffic received and forwarded has a positive relationship with throughput. Therefore, VLAN cannot raise network throughput, but it works to segment single large network domain into multi broadcast domains to achieve higher utilization of bandwidth with less traffic level.

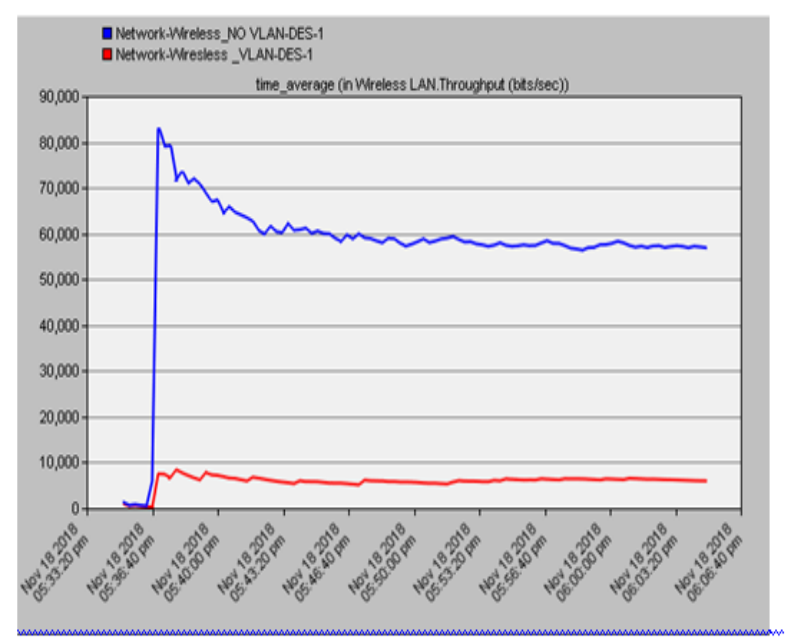

Figure 9. The wireless LAN throughput in the scenario 1 and scenario 2

Comparing the performance as shown in Figure 8 and Figure 9 give a clear picture about the selection of a VLAN over a wireless network that the delay has been reduced with the use of VLAN, whereas the throughput also reduced, which is the major drawback of using VLAN because in wireless networks, both short delay and high throughput are desirable. Therefore, we proposed an enhancement for the use of VLAN by employment various adhoc routing protocols such as AODV, OLSR, DSR, TORA and GRP and examine the impact of using these protocols to improve the wireless VLAN network throughput performance.

Considering the results of use adhoc routing protocols as shown in Figure 10 and Figure 11. We observe that delay can be further reduced depending on the routing protocol type. The delay for 
TORA is higher because of its route discovery process construction does not occur quickly and takes a lot of time discovering and deciding a route for data transfer leading to potential long delays. The DSR protocol uses cached routes and often it sends traffic on obsolete routes, which can cause retransmissions and cause increase delays. OLSR has the lowest delay because OLSR is a proactive routing protocol and routes in the network are always available whenever the application layer has a traffic to transmit. We conclude that OLSR exhibited very low delay in all scenarios. TORA had high delay, AODV and GRP had an improved wireless VLAN network delay.

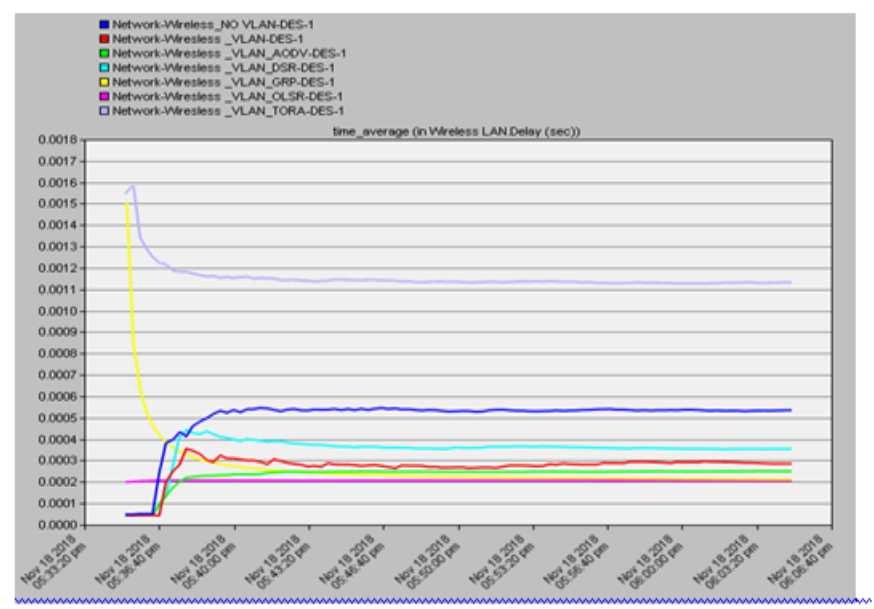

Figure 10. Comparison of Average delay in the wireless network for 20 Nodes

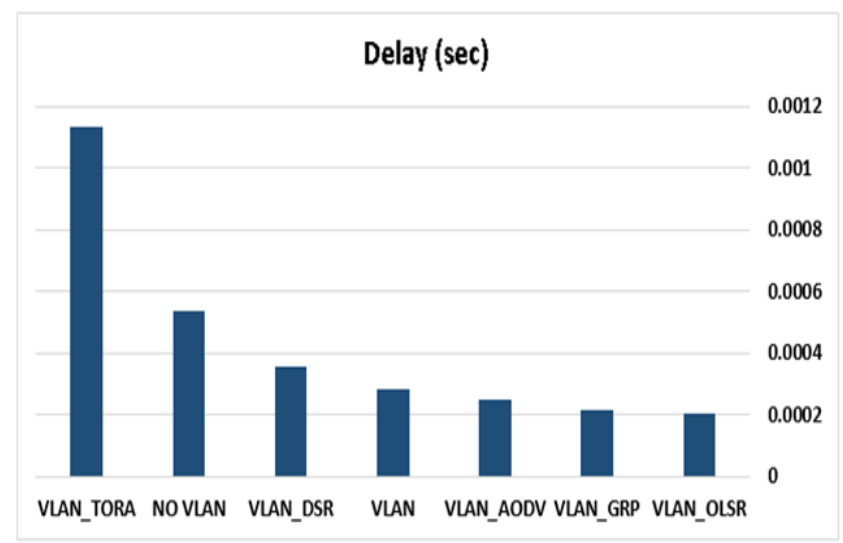

Figure 11. The wireless LAN delay for 20 Nodes

According to Figure 12 and Figure 13. We observe that an improvement in throughput can be achieved by employing routing protocol. AODV deserves the better performance with a small number of nodes followed by the DSR and OLSR. 


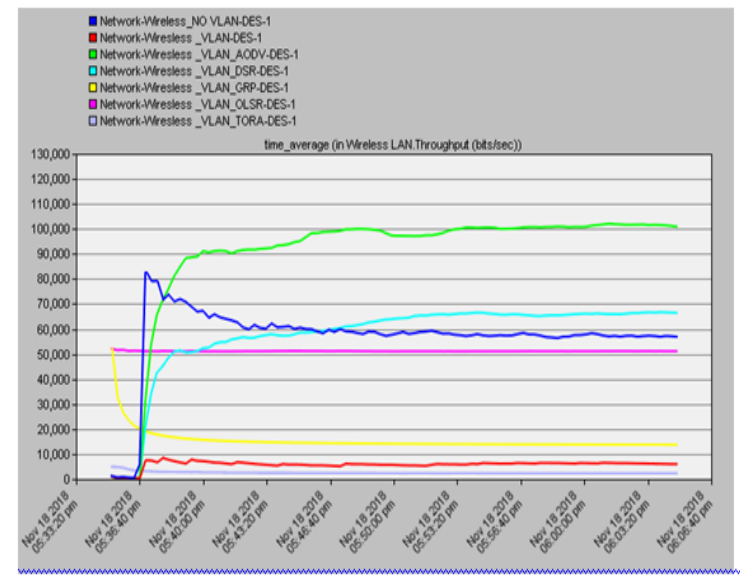

Figure 12. Comparison of throughput in the wireless network for 20 Nodes

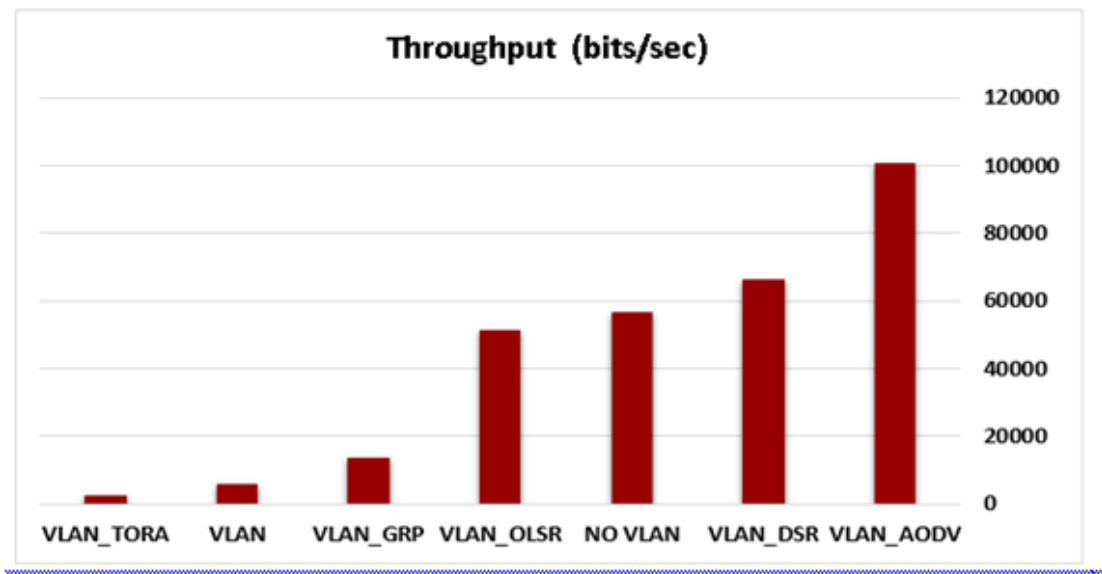

Figure 13. The wireless LAN throughput for 20 Nodes

To show the effect of the increase number of workstations on the performance improving accomplished by employing adhoc routing protocols on the wireless VLAN network, we return the simulation by increased number of nodes in each VLAN to be in order 20 nodes and 30 nodes.

In terms of throughput, with medium density network (40 Nodes) as seen in Figure 16 and Figure 17, AODV still performs better followed by DSR and OLSR. The rapid enhancement of AODV performance is clearly noticed when the number of nodes is increased. With regards to delay, still OLSR is performing well, as seen in Figure 14 and Figure 15. The overall results showed that the AODV gives higher throughput as the number of nodes is increased, whereas the OLSR gives the least delay. Furthermore, Regarding the AODV protocol, we notice an increase in delay when the network size increases, so we conclude that AODV is suitable for networks of low and medium load. 


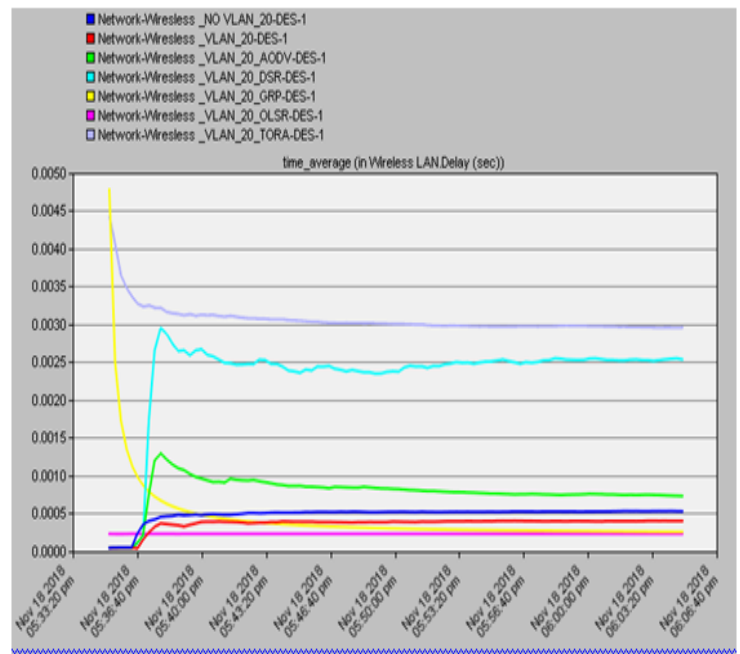

Figure 14. Comparison of Average delay in the wireless network for 40 Nodes

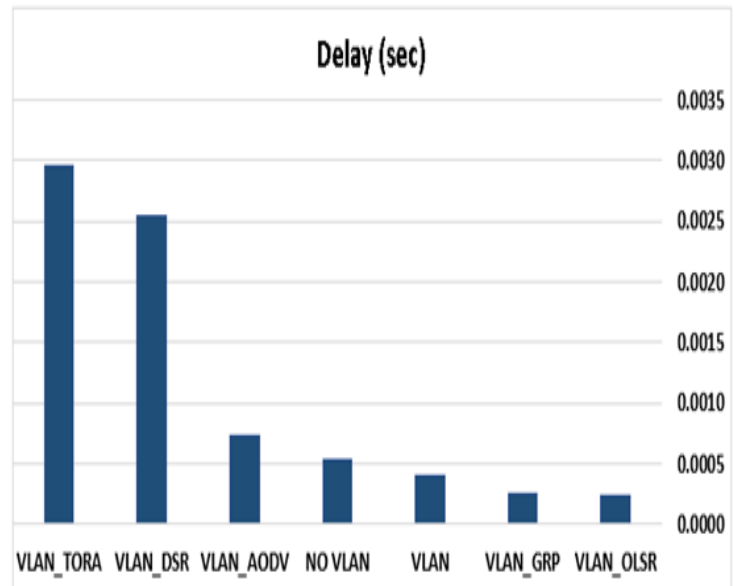

Figure 15. The wireless LAN delay for 40 Nodes

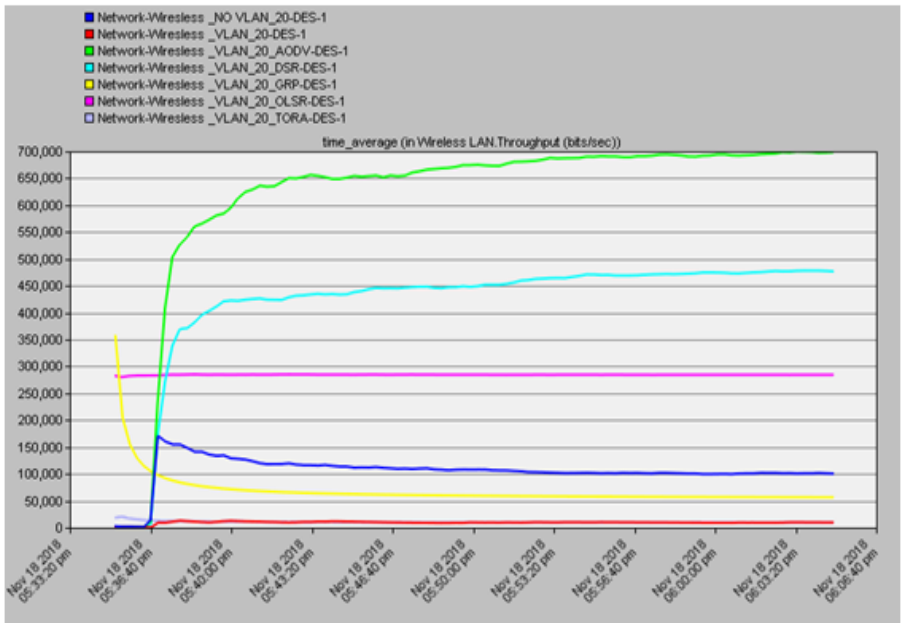

Figure 16. Comparison of throughput in the wireless network for 40 Nodes 


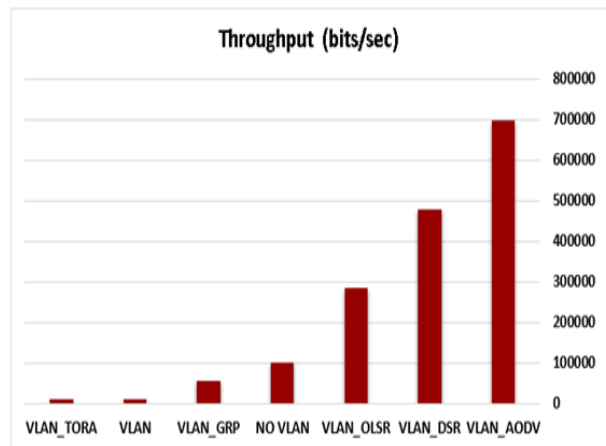

Figure 17. The wireless LAN throughput for 40 Nodes

In terms of throughput, with high density network (60 Nodes), AODV deserves the better performance followed by the DSR and OLSR as seen in Figure 20 and Figure 21. While, about the delay, still OLSR is performing well, as seen in Figure 18 and Figure 19. The decrease in AODV performance is clearly noticed as the number of nodes is increased.

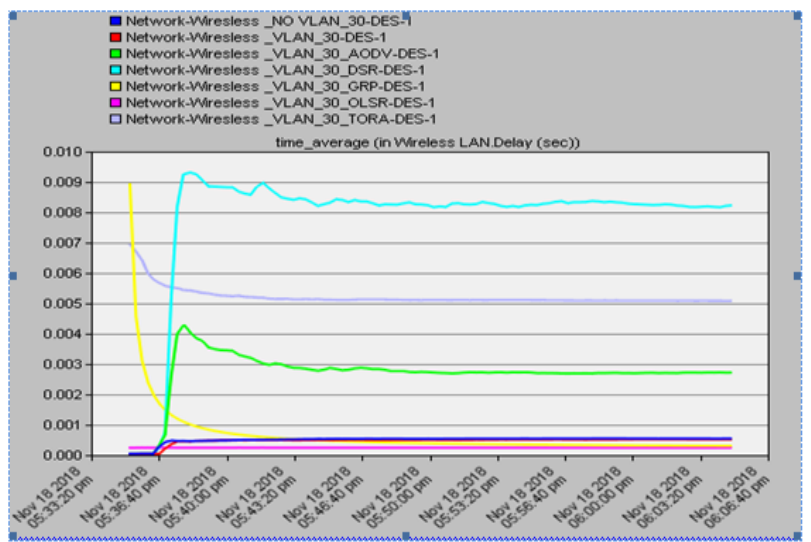

Figure 18. Comparison of Average delay in the wireless network for 60 Nodes

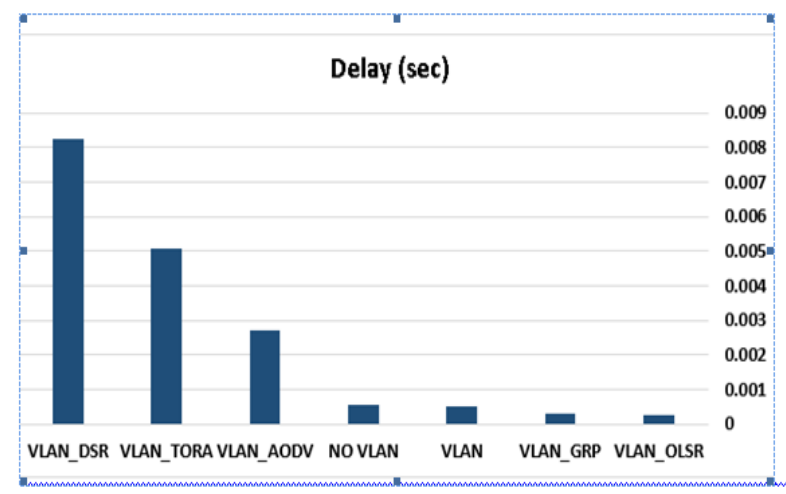

Figure 19. The wireless LAN delay for 60 Nodes 


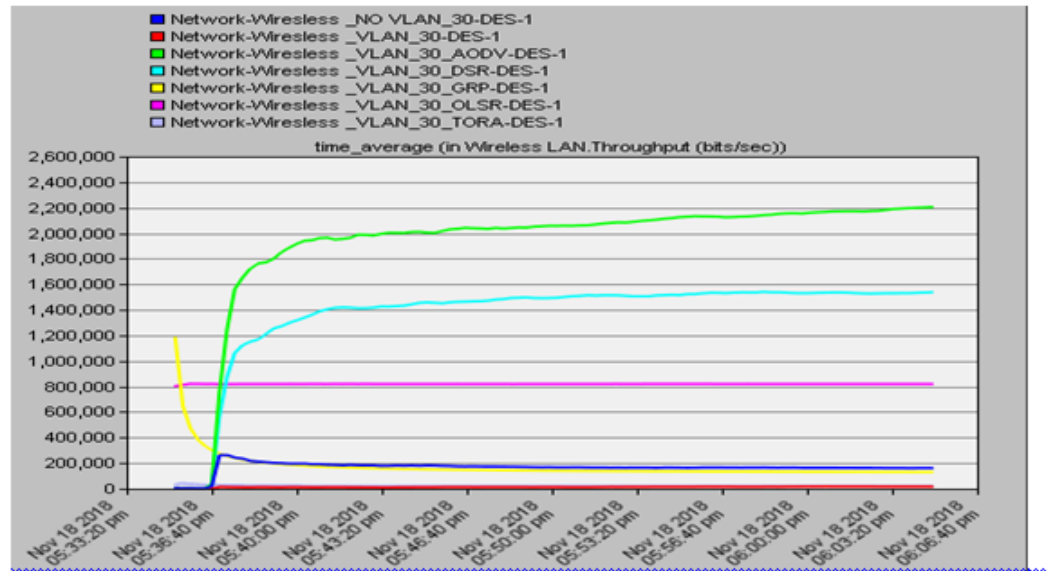

Figure 20. Comparison of throughput in the wireless network for 60 Nodes

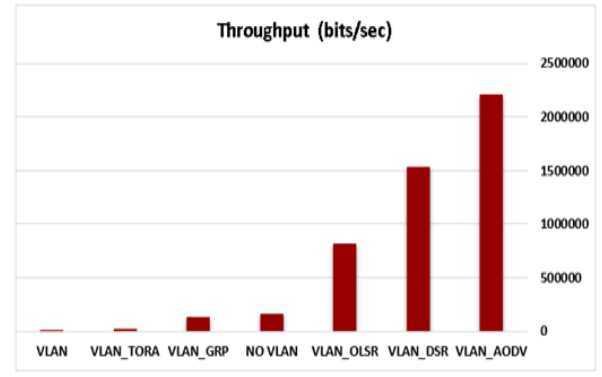

Figure 21. The wireless LAN throughput for 60 Nodes.

In conclusion, we observed that OLSR exhibits a very low delay in all scenarios while for the TORA protocol we note that it has a big delay, also with increase of traffic the DSR has a big delay and that because the size of traffic did the effect on delay. Regarding the AODV protocol, we notice an improvement in throughput when the network size increases, but on the other hand increase size of traffic did increase his delay. Finally, the results also show that the routing protocol type is an important factor to decide where throughput can improve more, or the delay can be improved more.

\section{Conclusions}

In this paper, we have presented employment of Virtual Local Area Network (VLAN) over a wireless LAN network with File Transfer Protocol (FTP) application and Hyper Text Transfer Protocol (HTTP) applications traffic using OPNET 14.5 simulator. Comparing performance has given a clear picture that implementing VLAN over a wireless network resulted in reducing both delay and throughput. While reducing delay is desirable, low throughput is a major drawback in wireless networks. To improve the wireless VLAN network throughput performance, we employed various adhoc routing protocols such as AODV, OLSR, DSR, TORA and GRP. The overall results showed that OLSR protocol performed better in terms of delay than others protocol regardless of network size, since OLSR is a proactive routing protocol and doesn't need to find routes to the destination. The reactive routing protocol AODV performs better in a low - density network, while performance degrades when the number of nodes is increased which concludes that it is more suitable for low-density network. However, while OLSR, DSR are performing well regarding throughput, the AODV is still dominant. As routing is desired for an appropriate operation of network, a network designer should select the 
suitable routing protocol that fits sufficiently the network's purpose. As a result, the selection of routing protocol type is an important factor to decide wither throughput or delay can improve more.

\section{ACKNOWLEDGEMENTS}

The authors would like to thank the Department of Computer Engineering, Jordan University of Science and Technology for the guidance and support for this research work. we would like to express our deep gratitude and thanks to the Dean of Computer and Information Technology for giving us helped in receiving various facilities and services from the university which allowed us to successfully implement this project.

\section{REFERENCES}

[1] Tambe, S. S., (2015) "Wireless technology in networks", International Journal of Scientific and Research Publications, Vol. 5, No. 7, pp1-3.

[2] Mittal, I., \& Anand, A., (2014) "WLan Architecture. International Journal of Computer Trends and Technology", Vol. 8, No. 3, pp. 148-151.

[3] Alimi, I. A., \& Mufutau, A. O., (2015) "Enhancement of network performance of an enterprises network with VLAN", American Journal of Mobile Systems, Applications and Services, Vol. 1, No. 2, pp 82-93.

[4] Elechi Onyekachi O., (2014) "Design and Simulation of Wireless Local Area Network for Administrative Office using OPNET Network Simulator: A Practical Approach", Information and Knowledge Management, Vol.4, No.10, pp. 27-34.

[5] Krupanek, B., \& Bogacz, R., (2016) "OPNET Modeler simulations of performance for multi nodes wireless systems", International Journal of Metrology and Quality Engineering, Vol. 7, No. 1, pp 105.

[6] H.S.Mewara , Mukesh Kumar Saini, (2014) " Performance Analysis of Access Point for IEEE 802.11g Wireless LAN Using Opnet Simulator" , International Journal of Advanced Engineering Research and Science (IJAERS), Vol.1, Issue.1, pp. 14-18.

[7] Sarah Ali Abdullah, (2016) "Simulation of Virtual LANs (VLANs) Using OPNET", IOSR Journal of Electronics and Communication Engineering (IOSR-JECE), Vol .11, Issue. 6, Ver. II, pp. 67-80.

[8] AL-Khaffaf, D. A. J., (2018) "Improving LAN Performance Based on IEEE802. 1Q VLAN Switching Techniques", Journal of University of Babylon, Vol. 26, No. 1, pp 286-297.

[9] Chokshi, R., \& Yu, C, (2007) "Study on VLAN in Wireless Networks", Technical Report.

[10] Ali, A. H., Abbas, A. N., \& Hassan, M. H., (2013) "Performance Evaluation of IEEE802. 11g WLANs Using OPNET Modeler", American Journal of Engineering Research (AJER), Vol. 2, Issue. 12, pp. 9-15.

[11] Meenakshi, Parneek Kaur, (2014) "Performance Metrics of WLAN for Different Applications using OPNET”, International Journal of P2P Network Trends and Technology (IJPTT), Vol. 4 Issue. 5.

[12] Alisa, Z. T., (2013) "Evaluating the performance of wireless network using OPNET modeler", International Journal of Computer Applications, Vol. 62, No. 13.

[13] Jayakumar, G., \& Ganapathy, G., (2007) "Performance comparison of mobile ad-hoc network routing protocol", International Journal of Computer Science and Network Security (IJCSNS), Vol. 7, No. 11, pp 77-84. 
[14] Manickam, P., Baskar, T. G., Girija, M., \& Manimegalai, D. D., (2011) "Performance comparisons of routing protocols in mobile ad hoc networks", arXiv preprint arXiv:1103.0658.

[15] Shrivastava, L., Bhadauria, S. S., \& Tomar, G. S., (2011) "Performance Evaluation of Routing Protocols in MANET with different traffic loads", In 2011 International Conference on Communication Systems and Network Technologies (pp. 13-16). IEEE.

[16] Maheswara, P. V., \& Naik, K. B., (2013) "Routing protocol performance issues and evaluation considerations in MANET", International Journal of Engineering Research and Applications (IJERA), Vol. 3, Issue. 4, pp.01-05.

[17] Elsadig, M. A., \& Fadlalla, Y. A. (2018) "Mobile Ad Hoc Network Routing Protocols: Performance Evaluation and Assessment", International Journal of Computing and Digital Systems, Vol. 7, No. 1, pp 59-66.

[18] Kadyamatimba, A., Mbougni, M., Helberg, Z. P. N. A., \& Dube, E., (2012) "Performance Evaluation of Routing Protocols in Mobile Ad Hoc Networks Using Http Traffic", DOI: 10.7763/IPEDR, Vol. 54, No. 14, pp 69-74.

[19] M. Quwaider, J. Rao, and S. Biswas, (2008) "Neighborhood route diffusion for packet salvaging in networks with high mobility", in IEEE International Performance, Computing and Communications Conference, pp. 168-175.

\section{AUTHORS}

Tareq Al-Khraishi received the B.Sc. In Computer Engineering, Al-Balqa Applied University, Jordan, 2003, and he is studying M.Sc. in Computer Engineering, Jordan University of Science and Technology (JUST), Jordan. His research interests focus on VLSI design and Nano-Technology in addition to wireless data networking, Cloud Computing and mobile ad hoc networks.

Muhannad Quwaider is an Associate Professor of computer engineering, college of Computer and Information Technology at Jordan University of Science and Technology (JUST). Dr. Quwaider earned his Ph.D. and M.S. at Michigan State University in East Lansing, USA, and his B.S. at Jordan University of Science and Technology in Irbid, Jordan. Prior to joining JUST in 2010, Dr. Quwaider was senior researcher in Networked Embedded and Wireless Systems (NeEWS) laboratory at the

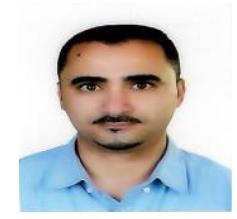
Electrical and Computer Engineering (ECE) Department of Michigan State University (MSU). Since 2012, Dr. Quwaider is Vice dean of Faculty of Computer and Information Technology, Jordan University of Science and Technology, Irbid, Jordan. His current research interests include the broad area of wireless data networking, Cloud Computing, Internet of Things, low-power network protocols, Big Data, application-specific sensor networks, wireless network security, mobile ad hoc networks, and body area network. 